\title{
Pendent Polymers of 1-Methyl-2-Oxo-4,5-Dicyanoimidazole and Their Electrochemical Properties
}

\author{
TAESEOK JANG, PAUL G. RASMUSSEN \\ Macromolecular Science and Engineering Center and Department of Chemistry, The University of Michigan, \\ Ann Arbor, Michigan 48109-1055
}

Received 23 May 2000; accepted 2 August 2000

\begin{abstract}
The electron accepting 1-methyl-4,5-dicyanoimidazole group was attached to vinyl polymers, via an alkoxy link, by nucleophilic aromatic substitution (NAS) of 1-methyl-2-fluoro-4,5-dicyanoimidazole (1) with poly(vinyl alcohol), or conventional polymerizations of vinyl monomers containing 1-methyl-2-oxo-4,5-dicyanoimidazole. The cyclic voltammetry (CV) studies show that monomeric and oligomeric model compounds are electrochemically quasi-reversible and the degree of reversibility decreases as dicyanoimidazoles become more proximate within a molecule. On the other hand, the polymers show much less reversible reduction waves at $-2.6 \sim-2.7 \mathrm{~V}$ vs $\mathrm{Ag} / \mathrm{Ag}^{+}$, suggesting that there are chemical reactions among the pendent groups reduced at relatively high potential. (C) 2000 John Wiley \& Sons, Inc. J Polym Sci A: Polym Chem 38: 3828-3838, 2000
\end{abstract}

Keywords: cyclic voltammetry; nucleophilic aromatic substitution; poly(vinyl alcohol); 4,5-dicyanoimidazoles; quasi-reversible

\section{INTRODUCTION}

Many organic compounds with cyano groups are known as electron acceptors. Representative examples that are particularly well known include tetracyanoquinodimethane (TCNQ) and tetracyanoethylene (TCNE). These materials form charge transfer complexes with electron donors such as tetrathiafulvalene (TTF), and give rise to conductivity as high as $500 \mathrm{~S} / \mathrm{cm}^{1}{ }^{1}$ In an effort to make organic polymer acceptors, there have been a few, partially successful, efforts to functionalize TCNQ into polymer backbone. ${ }^{2}$ These polymer acceptors have the potential to be used as the complementary reducible parts of oxidizable polymeric materials in applications such as polymer batteries and electrochemical capacitors. ${ }^{3}$ Whereas many oxidized polymers have been developed and

Correspondence to: P. G. Rasmussen (E-mail: pgrasmsn@ umich.edu)

Journal of Polymer Science: Part A: Polymer Chemistry, Vol. 38, 3828-3838 (2000) (๑) 2000 John Wiley \& Sons, Inc. studied since the early $1980 \mathrm{~s},{ }^{4}$ less effort has gone into preparing polymeric electron accepting materials. $^{5}$

We have been investigating the chemistry and properties of cyanoimidazole compounds as electron acceptors. ${ }^{6}$ Cyclic voltammetry (CV) is a good way to determine the electron-accepting strength of compounds, and our previous work showed that the reduction potentials of cyanoimidazole compounds lie between strong acceptors like TCNQ and homoaromatic cyano compounds like benzonitrile. The 1-methyl-4,5-dicyano-midazoles containing a dialkylamino group at the 2 position show electrochemical reversibility, which is a prerequisite for applications. We previously reported the synthesis and electrochemical properties of oligomers and polymers containing 1-methyl-4,5dicyanoimidazole, with a dialkylamino link, as a pendent group. ${ }^{7}$

As part of our search for electron accepting materials based on dicyanoimidazole, we report here the synthesis and cyclic voltammetry data for a series of 2-oxo derivatives of 1-methyl-4,5- 
dicyanoimidazole and the results of incorporation of the 1-methyl-4,5-dicyanoimidazole pendent group, via an alkoxy link, into polymer main chains.

\section{EXPERIMENTAL}

\section{Materials and Procedures}

1-Methyl-2-fluoro-4,5-dicyanoimidazole (1) was prepared as previously described ${ }^{8}$ by transfluorination of 1-methyl-2-bromo-4,5-dicyanoimidazole (2) using potassium fluoride and a catalytic amount of 18-crown-6 in diglyme. Other reagents were purchased from Aldrich Chemical Co., and used as received. Tetrahydrofuran (THF) was distilled from sodium benzophenone ketyl. Dimethylsulfoxide (DMSO) was distilled over $\mathrm{BaO}$ under vacuum $(0.1-1.0 \mathrm{mmHg})$ and stored over $4 \AA$ molecular sieves.

Thin-layer chromatography (TLC) was performed using Eastman Kodak silica gel sheets containing fluorescent indicator. Infrared (IR) spectra were obtained using a Nicolet 5-DX FTIR spectrophotometer. ${ }^{1} \mathrm{H}$ and ${ }^{13} \mathrm{C}$ NMR spectra were recorded on a Bruker AM-360 or AM-300 spectrometer. Nominal mass spectra and high resolution mass spectra were recorded on a VG analytical model 70-250S mass spectrometer. Gel permeation chromatographic (GPC) analyses were carried out using three $7.8 \times 300 \mathrm{~mm}$ Styrogenl columns on a Waters 510 GPC system. THF was used as the eluent. Molecular weights were calibrated with narrow molecular weight polystyrene standards.

\section{Cyclic Voltammetry}

Cyclic voltammetry was performed using a Princeton Applied Research (PAR) potentiostat/ galvanostat, model 173, a PAR universal programmer, model 175 and a PAR digital coulometer, model 179. Acetonitrile was distilled over $\mathrm{CaH}_{2}$ immediately prior to use. The electrolyte solution was $0.1 \mathrm{M} \mathrm{Et}_{4} \mathrm{NBF}_{4}$ in acetonitrile. Tetraethylammonium tetrafluoroborate $\left(\mathrm{Et}_{4} \mathrm{NBF}_{4}\right.$, 99\%, Aldrich) was recrystallized three times and dried in vacuum. Samples were $5-10 \mathrm{~m} M$ in the electrolyte solution. The reference solution was $0.01 M$ silver nitrate in the electrolyte solution and the reference electrode was a silver wire. The working electrode was a platinum wire and the counter electrode was a platinum foil. Dry nitro- gen gas was passed through sample solutions for 30 min to remove oxygen.

\section{Synthesis of Alkoxy and Aryloxy derivatives}

\section{General Procedure}

A 50-mL round-bottomed flask, equipped with a reflux condenser and a magnetic stirring bar, was charged with equimolar 1-methyl-2-fluoro-4,5-dicyano-imidazole (1) and an alcohol or phenol in THF and one equivalent of potassium carbonate. The mixture was refluxed for 1 to $18 \mathrm{~h}$. The mixture was cooled, and the solvent removed using a rotary evaporator. The concentrated mixture was poured into excess water. If the product precipitated, the solid obtained was recrystallized from various solvents. Otherwise, the aqueous solution was extracted with ethyl acetate three times. The ethyl acetate layer was dried over anhydrous magnesium sulfate and the ethyl acetate was removed under vacuum. The remaining product was purified by column chromatography, if necessary.

\section{1-Methyl-2-ethoxy-4,5-dicyanoimidazole (2)}

The reaction was carried out using $1(0.10 \mathrm{~g}, 6.7$ $\mathrm{mmol})$ and absolute ethanol $(10 \mathrm{~mL})$ without THF. The product was recrystallized from water/ ethanol $(1 / 1 \mathrm{v} / \mathrm{v})$ mixture to give white needles ( $0.074 \mathrm{~g}, 63 \%$ yield). m.p. $80-81{ }^{\circ} \mathrm{C}$; TLC $\mathrm{R}_{\mathrm{f}} 0.65$ (in EtOAc); MS (EI/70eV) $\mathrm{m} / z: 176(25, \mathrm{M}+), 161$ (3), 148 (100), 121 (13), 106 (7), 93 (8), 68 (30), 53 (9), 41 (7); FTIR (KBr) 2997, 2237, 1588, 1489, $1392,1019 \mathrm{~cm}^{-1} ;{ }^{1} \mathrm{H} \mathrm{NMR}\left(\mathrm{CDCl}_{3}\right) \delta(\mathrm{ppm}) 4.52(\mathrm{q}$, $\left.2 \mathrm{H}, \mathrm{CH}_{2}, J=7.2 \mathrm{~Hz}\right), 3.56\left(\mathrm{~s}, 3 \mathrm{H}, \mathrm{ArCH}_{3}\right), 1.45$ (t, $\left.3 \mathrm{H}, \mathrm{CH}_{2} \mathrm{CH}_{3}, J=7.2 \mathrm{~Hz}\right) ;{ }^{13} \mathrm{C} \operatorname{NMR}\left(\mathrm{CDCl}_{3}\right)$ $\delta(\mathrm{ppm}) 154.1,118.4,112.2,109.2,108.7,68.3$, 30.7, 14.5; ANal. Calcd for $\mathrm{C}_{8} \mathrm{H}_{8} \mathrm{~N}_{4} \mathrm{O}: \mathrm{C}, 54.54 ; \mathrm{H}$, 4.58. Found: C, 54.46; H, 4.59.

\section{1-Methyl-2-trifluoroethoxy-4,5- dicyanoimidazole (3)}

The reaction was carried out using $\mathbf{1}(0.20 \mathrm{~g}, 1.3$ $\mathrm{mmol})$ and 2,2,2-trifluoro-ethanol $(0.97 \mathrm{~mL}, 13$ mmol). Oily liquid ( $0.305 \mathrm{~g}, 99 \%$ yield). TLC $R_{\mathrm{f}}$ 0.68 (in EtOAc); MS (EI/70eV) $m / z: 230(61, \mathrm{M}+$ ), 211 (5), 161 (10), 147 (100), 120 (28), 106 (4), 95 (12), 83 (16), 67 (75), 54 (8), 33 (30), 15 (68); FTIR (KBr window) 2963, 2241, 2235, 1730, 1556, 1502, 1453, 1409, 1369, 1274, 1239, 1168, 1066, 1038, 965, $893 \mathrm{~cm}^{-1} ;{ }^{1} \mathrm{H}$ NMR $\left(\mathrm{CDCl}_{3}\right) \delta(\mathrm{ppm}) 4.81(\mathrm{q}$, $\left.2 \mathrm{H}, \mathrm{CH}_{2}, J=7.9 \mathrm{~Hz}\right), 3.65\left(\mathrm{~s}, 3 \mathrm{H}, \mathrm{CH}_{3}\right) ;{ }^{13} \mathrm{C} \mathrm{NMR}$ 
$\left(\mathrm{CDCl}_{3}\right) \delta(\mathrm{ppm}) 152.3,122.3(\mathrm{~d}, J=275 \mathrm{~Hz})$, $118.3,111.6,110.5,108.0,67.0$ (q, $J=37.6 \mathrm{~Hz}$ ), 31.20; ANAL. Calcd for $\mathrm{C}_{8} \mathrm{H}_{5} \mathrm{~N}_{4} \mathrm{OF}_{3}: \mathrm{C}, 41.75 ; \mathrm{H}$, 2.19. Found: C, 41.78; H, 2.26.

\section{1-Methyl-2-propargyloxy-4,5-dicyanoimidazole (4)}

The reaction was carried out using $\mathbf{1}(2.00 \mathrm{~g}, 13.3$ mmol) and propargyl alcohol (1.16 mL, 19.9 mmol). Yellow solid was recrystallized from hexane/toluene (9/1) to give a white powder $(1.19 \mathrm{~g}$, $48.0 \%$ yield), m.p. $72-73{ }^{\circ} \mathrm{C}$. TLC $\mathrm{R}_{\mathrm{f}} 0.67$ (in EtOAc); MS (EI/70eV) m/z: $186(22, \mathrm{M}+), 157$ (4), 147 (5), 132 (4), 121 (14), 107 (3), 93 (5), 77 (3), 67 (25), 52 (5), 39 (100\%), 15 (26); FTIR (KBr) 3274, 2237, 2134, 1550, 1500, 1449, 1358, 1324, 1179, 1006, 961, 721, $676 \mathrm{~cm}^{-1}$; ${ }^{1} \mathrm{H}$ NMR $\left(\mathrm{CDCl}_{3}\right)$ $\delta(\mathrm{ppm}) 5.05\left(\mathrm{~s}, 2 \mathrm{H}, \mathrm{CH}_{2}\right), 3.59\left(\mathrm{~s}, 3 \mathrm{H}, \mathrm{ArCH}_{3}\right)$, $2.62(\mathrm{~s}, 1 \mathrm{H},+\mathrm{CH}) ;{ }^{13} \mathrm{C}$ NMR $\left(\mathrm{CDCl}_{3}\right) \delta(\mathrm{ppm})$ $153.6,118.3,111.9,110.1,108.4,77.5,76.2$, 59.2, 31.0. HRMS Found: 186.0532 Calcd for $\mathrm{C}_{9} \mathrm{H}_{6} \mathrm{~N}_{4} \mathrm{O}_{1}: 186.0541$.

\section{1-Methyl-2-phenoxy-4,5-dicyanoimidazole (5)}

The reaction was carried out using 1 ( $0.200 \mathrm{~g}, 1.33$ $\mathrm{mmol})$ and phenol $(0.125 \mathrm{~g}, 1.33 \mathrm{mmol})$. The product was recrystallized from ethanol to give white crystals $(0.212$ g, $71.0 \%)$. m.p. $145-146{ }^{\circ} \mathrm{C}$; MS (EI/70eV) $m / z: 224(66, \mathrm{M}+), 207$ (3), 195 (13), 181 (2), 166 (3), 147 (3), 129 (13), 121 (8), 103 (4), 91 (68), 77 (100), 67 (17), 51 (72), 39 (10); FTIR (KBr) 2238, 1541, 1496, 1487, 1455, 1407, 1327, 1204, 1192, 1153, 760, $695 \mathrm{~cm}^{-1} ;{ }^{1} \mathrm{H}$ NMR $\left(\mathrm{CDCl}_{3}\right)$ $\delta(\mathrm{ppm}) 7.45(\mathrm{~d}, 2 \mathrm{H}, o-\mathrm{Ph} \mathrm{CH}, J=8 \mathrm{~Hz}), 7.29(\mathrm{~m}$, $3 \mathrm{H}, m$ - and $p$ - $\mathrm{Ph} \mathrm{CH}), 3.76\left(\mathrm{~s}, 3 \mathrm{H}, \mathrm{ArCH}_{3}\right) ;{ }^{13} \mathrm{C}$ NMR $\left(\mathrm{CDCl}_{3}\right) \delta(\mathrm{ppm}) 152.7,152.6,130.2,126.8$, $119.9,118.6,111.8,110.0,108.3,31.4$; ANaL. Calcd for $\mathrm{C}_{12} \mathrm{H}_{8} \mathrm{~N}_{4} \mathrm{O}: \mathrm{C}, 64.28 ; \mathrm{H}, 3.60$. Found: C, $64.20 ; \mathrm{H}, 3.63$.

\section{1-Methyl-2-p-nitrophenoxy-4,5- dicyanoimidazole (6)}

The reaction was carried out using $\mathbf{1}(0.200 \mathrm{~g}, 1.33$ $\mathrm{mmol})$ and $p$-nitrophenol (0.186 g, $1.33 \mathrm{mmol})$. The product was recrystallized from acetone/ethanol $(1 / 1 \mathrm{v} / \mathrm{v})$ mixture to give white crystals $(0.231$ g, 64\% yield), m.p. $208-210{ }^{\circ} \mathrm{C}$; FTIR (KBr) 2246 , 2238, 1618, 1594, 1529, 1523, 1497, 1365, 1346, $1322 \mathrm{~cm}^{-1} ;{ }^{1} \mathrm{H}$ NMR (DMSO-d 6 ) $\delta(\mathrm{ppm}) 8.37(\mathrm{~d}$, $2 \mathrm{H}, \mathrm{m}-\mathrm{Ph} \mathrm{CH}, J=8.9 \mathrm{~Hz}$ ), 7.68 (d, 2H, o-Ph CH, $J=8.9 \mathrm{~Hz}), 3.72\left(\mathrm{~s}, 3 \mathrm{H}, \mathrm{ArCH}_{3}\right) ;{ }^{13} \mathrm{C} \mathrm{NMR}$ $\left(\right.$ DMSO$\left._{-} \mathrm{d}_{6}\right) \quad \delta(\mathrm{ppm}) \quad 157.3,150.8,144.9,125.9$, 1205.5, 116.3, 112.2, 110.9, 108.6, 31.8; ANAL.
Calcd for $\mathrm{C}_{12} \mathrm{H}_{7} \mathrm{~N}_{5} \mathrm{O}_{3}$ : C, 53.54; $\mathrm{H}, 2.62$. Found: C, $53.45 ; \mathrm{H}, 2.66$.

\section{1,4-Bis(1-methyl-4,5-dicyano-2- imidazolyloxy)benzene (7)}

The reaction was carried out using $\mathbf{1}(0.200 \mathrm{~g}, 1.33$ $\mathrm{mmol})$ and hydroquinone $(0.073 \mathrm{~g}, 0.67 \mathrm{mmol})$. The product was recrystallized from ethanol (0.221 g, 90\% yield), m.p. $215-219{ }^{\circ} \mathrm{C}$; TLC $\mathrm{R}_{\mathrm{f}} 0.61$ (in EtOAc); FTIR (KBr) 2231, 1538, 1491, 1407, 1326, 1204, 1191, $1158 \mathrm{~cm}^{-1} ;{ }^{1} \mathrm{H}$ NMR (DMSO-d ${ }_{6}$ ) $\delta(\mathrm{ppm}) 7.45(\mathrm{~s}, 4 \mathrm{H}$, phenyl $\mathrm{CH}), 3.71(\mathrm{~s}, 6 \mathrm{H}$, $\mathrm{ArCH}_{3}$ ); ANAL. Calcd for $\mathrm{C}_{18} \mathrm{H}_{10} \mathrm{~N}_{8} \mathrm{O}_{2}: \mathrm{C}, 58.38 ; \mathrm{H}$, 2.72. Found: C, 57.29; H, 4.21.

\section{2,2-Bis[p-(1-methyl-4,5-dicyano-2- imidazolyloxy) phenyl]propane (8)}

The reaction was carried out using $\mathbf{1}(0.200 \mathrm{~g}, 1.33$ mmol) and bisphenol A (0.152 g, $0.667 \mathrm{mmole})$. The product was recrystallized from ethanol (0.301 g, 93\% yield), m.p. $210-211{ }^{\circ} \mathrm{C}$; TLC $\mathrm{R}_{\mathrm{f}}$ 0.67 (in EtOAc); FTIR (KBr) 2970, 2234, 1541, 1502, 1489, 1404, 1326, 1240, 1209, $1178 \mathrm{~cm}^{-1}$; ${ }^{1} \mathrm{H}$ NMR $\left(\mathrm{DMSO}_{-} \mathrm{d}_{6}\right) \delta(\mathrm{ppm}) 7.29$ (q, 8H, phenyl $\mathrm{CH}), 3.69\left(\mathrm{~s}, 6 \mathrm{H}, \mathrm{ArCH}_{3}\right), 1.68\left(\mathrm{~s}, 6 \mathrm{H}, \mathrm{C}^{-\mathrm{CH}_{3}}\right) ;{ }^{13} \mathrm{C}$ NMR (DMSO- $\left.\mathrm{d}_{6}\right) \delta(\mathrm{ppm}) 152.3,150.9,147.9$, 128.0, 119.4, 116.4, 112.4, 110.4, 108.8, 42.1, 31.5, 30.4; ANAL. Calcd for $\mathrm{C}_{27} \mathrm{H}_{20} \mathrm{~N}_{8} \mathrm{O}_{2}: \mathrm{C}, 66.39 ; \mathrm{H}$, 4.13. Found: C, 66.03; H, 4.36.

\section{Oligomeric Alkoxy Model Compounds}

\section{General Procedure}

Into a 50-mL round-bottomed flask equipped with nitrogen inlet and magnetic stirring bar were charged equimolar 1, diol (or polyol), and 1 equivalent of potassium carbonate. The mixture was dissolved in DMSO (2-3 mL) to make a 20-30 wt $\%$ solution. The solution was stirred at room temperature for $24-48 \mathrm{~h}$, and then poured into ice water $(30 \mathrm{~mL})$ to give a white precipitate. This crude product was collected by filtration and recrystallized from toluene or toluene/hexane.

\section{1,2-Bis(1-methyl-4,5-dicyano-2- imidazolyloxy)ethane (9)}

The reaction was carried out using $\mathbf{1}(0.316 \mathrm{~g}, 2.11$ $\mathrm{mmol})$ and ethylene glycol $(0.054 \mathrm{~g}, 0.87 \mathrm{mmol})$. The pale yellow solid was recrystallized from toluene to give a white powder $(0.155 \mathrm{~g}, 55 \%$ yield $)$, m.p. $158-160{ }^{\circ} \mathrm{C}$. TLC $\mathrm{R}_{\mathrm{f}} 0.48$ (in EtOAc); FTIR (KBr) 2233, 2224, 1551, 1496, 1487, 1455, 1406, 
1367, 1348, 1326, 1205, 1180, $1059 \mathrm{~cm}^{-1} ;{ }^{1} \mathrm{H}$ NMR (DMSO-d $\left.{ }_{6}\right) \delta(\mathrm{ppm}) 4.79\left(\mathrm{~s}, 4 \mathrm{H}, \mathrm{OCH}_{2}\right), 3.51$ $\left(\mathrm{s}, 6 \mathrm{H}, \mathrm{ArCH}_{3}\right) ;{ }^{13} \mathrm{C}$ NMR (DMSO-d 6 ) $\delta(\mathrm{ppm})$ 153.5, 116.3, 112.5, 109.9, 108.8, 69.2, 30.9; ANAL. Calcd for $\mathrm{C}_{14} \mathrm{H}_{10} \mathrm{~N}_{8} \mathrm{O}_{2}$ : C, 52.18; $\mathrm{H}, 3.13$. Found: C, 52.53; H, 3.19.

\section{1,3-Bis(1-methyl-4,5-dicyano-2- imidazolyloxy) propane (10)}

The reaction was carried out using $\mathbf{1}(0.189 \mathrm{~g}, 1.26$ $\mathrm{mmol}$ ) and 1,3-propanediol (0.043 g, $0.57 \mathrm{mmol})$. The off-white solid was collected and recrystallized from toluene to give a white powder $(0.051 \mathrm{~g}$, $27 \%$ yield). m.p. $132-133{ }^{\circ} \mathrm{C}$. TLC $\mathrm{R}_{\mathrm{f}} 0.50$ (in EtOAc); FTIR (KBr) 2237, 1559, 1492, 1469, 1382, 1376, 1340, 1318, 1178, 1052, 1028, 981, 964 $\mathrm{cm}^{-1} ;{ }^{1} \mathrm{H}$ NMR (acetone- $\mathrm{d}_{6}$ ) $\delta(\mathrm{ppm}) 4.68(\mathrm{t}, 4 \mathrm{H}$, $\mathrm{OCH}_{2}, J=6 \mathrm{~Hz}$ ), $3.65\left(\mathrm{~s}, 6 \mathrm{H}, \mathrm{CH}_{3}\right), 2.39$ (quintet, $2 \mathrm{H}, \quad \beta \mathrm{CH}_{2}, \quad J=6 \mathrm{~Hz}$; ANAL. Calcd for $\mathrm{C}_{15} \mathrm{H}_{12} \mathrm{~N}_{8} \mathrm{O}_{2}$ : C, 53.57; H, 3.60. Found: C, 53.22; $\mathrm{H}, 3.42$.

\section{1,4-Bis(1-methyl-4,5-dicyano-2- imidazolyloxy)butane (11)}

The reaction was carried out using 1 ( $0.321 \mathrm{~g}, 2.14$ mmol) and 1,4-butanediol (0.087 g, $0.97 \mathrm{mmol}$ ). The pale yellow solid collected was recrystallized from toluene to give a white powder $(0.140 \mathrm{~g}, 41 \%$ yield), m.p. $154-156{ }^{\circ} \mathrm{C}$. TLC $\mathrm{R}_{\mathrm{f}} 0.55$ (in EtOAc); FTIR (KBr) 2967, 2237, 1561, 1491, 1465, 1447, $1409,1365,1322,1200,1178,1054,1036,966$ $\mathrm{cm}^{-1} ;{ }^{1} \mathrm{H}$ NMR (DMSO-d 6 ) $\delta(\mathrm{ppm}) 4.47(\mathrm{t}, 4 \mathrm{H}$, $\left.\mathrm{OCH}_{2}, J=6 \mathrm{~Hz}\right), 3.51\left(\mathrm{~s}, 6 \mathrm{H}, \mathrm{ArCH}_{3}\right), 1.89$ (t, $4 \mathrm{H}$, $\left.\beta \mathrm{CH}_{2}, J=6 \mathrm{~Hz}\right) ;{ }^{13} \mathrm{C}$ NMR (DMSO-d 6 ) $\delta(\mathrm{ppm})$ 153.8, 116.3, 112.6, 109.6, 109.0, 71.2, 30.8, 24.5; ANAL. Calcd for $\mathrm{C}_{16} \mathrm{H}_{14} \mathrm{~N}_{8} \mathrm{O}_{2}$ : C, 54.85; H, 4.03. Found: C, 55.11; H, 4.16.

\section{1,6-Bis(1-methyl-4,5-dicyano-2- imidazolyloxy)hexane (12)}

The reaction was carried out using 1 ( $0.326 \mathrm{~g}, 2.17$ $\mathrm{mmol}$ ) and 1,6-hexanediol (0.116 g, $0.983 \mathrm{mmol})$. The pale yellow solid was collected and recrystallized from toluene to give white powder $(0.109 \mathrm{~g}$, $30 \%$ yield). m.p. $106-109{ }^{\circ} \mathrm{C}$. TLC $\mathrm{R}_{\mathrm{f}} 0.66$ (in EtOAc); FTIR (KBr) 2950, 2235, 1559, 1492, 1474, $1459,1378,1355,1317,1175,1070,1039,984$, $723,720 \mathrm{~cm}^{-1} ;{ }^{1} \mathrm{H}$ NMR (acetone- $\left.\mathrm{d}_{6}\right) \delta(\mathrm{ppm}) 4.49$ (t, $\left.4 \mathrm{H}, \mathrm{OCH}_{2}, J=6.5 \mathrm{~Hz}\right), 3.64\left(\mathrm{~s}, 6 \mathrm{H}, \mathrm{ArCH}_{3}\right)$, $1.87\left(\mathrm{~m}, 4 \mathrm{H}, \beta \mathrm{CH}_{2}\right), 1.56\left(\mathrm{~m}, 4 \mathrm{H}, \gamma \mathrm{CH}_{2}\right) ;{ }^{13} \mathrm{C}$ NMR (acetone- $\mathrm{d}_{6}$ ) $\delta(\mathrm{ppm}) 155.3,118.3,113.3$, 110.5, 109.5, 72.7, 31.3, 29.3, 25.9; ANAL. Calcd for
$\mathrm{C}_{18} \mathrm{H}_{18} \mathrm{~N}_{8} \mathrm{O}_{2}$ : C, 57.14; H, 4.79. Found: C, 57.27; $\mathrm{H}, 4.84$.

\section{1,1,1-Tris[(1-methyl-4,5-dicyano-2- imidazolyl)oxomethyl]ethane (13)}

The reaction was carried out using $\mathbf{1}(0.338 \mathrm{~g}$, $2.25 \mathrm{mmol}$ ) and 1,1,1-tris(hydroxymethyl)ethane $(0.080 \mathrm{~g}, 0.67 \mathrm{mmol})$. The pale yellow solid was recrystallized from toluene/hexane $(1 / 3 \mathrm{v} / \mathrm{v})$ to give white powder $(0.230 \mathrm{~g}, 67 \%$ yield $)$. m.p. 100-105 ${ }^{\circ} \mathrm{C}$ dec. HRMS Calcd for $[\mathrm{M}+\mathrm{H}]^{+}$ $\mathrm{C}_{23} \mathrm{H}_{19} \mathrm{~N}_{12} \mathrm{O}_{3}$ 511.1703. Found 511.1695.

\section{Tetrakis[(1-methyl-4,5-dicyano-2- imidazolyl)oxomethyl]methane (14)}

The reaction was carried out using 1 ( $0.320 \mathrm{~g}, 2.13$ $\mathrm{mmol})$ and pentaerythritol $(0.066 \mathrm{~g}, 0.49 \mathrm{mmol})$. The solid was collected and recrystallized from acetone/ethanol $(2 / 1 \mathrm{v} / \mathrm{v})$ mixture to give a white powder $(0.163 \mathrm{~g}, 51 \%$ yield $)$, m.p. $254-256{ }^{\circ} \mathrm{C}$. TLC $\mathrm{R}_{\mathrm{f}}$ (in EtOAc); FTIR (KBr) 2237, 1552, 1459, 1392，1365，1324，1201，1180，1055，999, 719 $\mathrm{cm}^{-1} ;{ }^{1} \mathrm{H}$ NMR (DMSO-d $\left.{ }_{6}\right) \delta(\mathrm{ppm}) 4.75(\mathrm{~s}, 8 \mathrm{H}$, $\left.\mathrm{CH}_{2}\right), 3.56\left(\mathrm{~s}, 12 \mathrm{H}, \mathrm{CH}_{3}\right) ;{ }^{13} \mathrm{C}$ NMR $\left(\mathrm{DMSO}_{-} \mathrm{d}_{6}\right)$ $\delta(\mathrm{ppm}) 153.2,116.1,112.3,109.8,108.7,68.1$, 44.0, 30.9; ANAL. Calcd for $\mathrm{C}_{29} \mathrm{H}_{20} \mathrm{~N}_{16} \mathrm{O}_{4}$ : C, 53.05; H, 3.07. Found: C, 53.13; H, 3.26.

\section{Pendent Polymers with an Oxygen Link}

\section{Substitution Reaction to Poly(vinyl alcohol) (15)}

Into a solution of poly(vinyl alcohol) $\left(M_{w}=89-98\right.$ $\mathrm{kg} / \mathrm{mol}, 99 \%$ hydrolyzed, Aldrich) $(0.50 \mathrm{~g}, 11 \mathrm{mmol}$ repeating unit) in DMSO (5 mL) was added 1 $(2.56 \mathrm{~g}, 17.1 \mathrm{mmol})$. The mixture was stirred at 90 ${ }^{\circ} \mathrm{C}$ for $48 \mathrm{~h}$, cooled to room temperature, and poured into methanol $(100 \mathrm{~mL})$. The white precipitate was washed with ether, and dried in a vacuum oven. $(0.428 \mathrm{~g}, 22 \%$ yield). FTIR ( $\mathrm{KBr})$ 3300(br), 2943, 2918, 2235, 1558, 1489, 1436, 1419, 1375, 1325, 1094, $1021 \mathrm{~cm}^{-1} .{ }^{1} \mathrm{H}$ NMR $\left(\right.$ DMSO $\left._{6}\right) \delta(\mathrm{ppm}) 4.45(\mathrm{~m}, 1 \mathrm{H}, \mathrm{OCH}), 3.82(\mathrm{~s}, 0.8$ $\times 1 \mathrm{H}, \mathrm{OH}), 3.48\left(\mathrm{~s}, 0.2 \times 3 \mathrm{H}, \mathrm{ArCH}_{3}\right), 1.39(\mathrm{~d}, 2 \mathrm{H}$, $\left.\mathrm{CH}_{2}\right)$; ANAL. Calcd for $\left(\mathrm{C}_{2} \mathrm{H}_{4} \mathrm{O}\right)_{0.8}\left(\mathrm{C}_{8} \mathrm{H}_{6} \mathrm{~N}_{4} \mathrm{O}\right)_{0.2^{-}}$ $\left(\mathrm{H}_{2} \mathrm{O}\right)_{0.25}$ : C, 51.54; H, 6.62. Found: C, 51.54; $\mathrm{H}, 6.89$.

\section{2-(1-Methyl-4,5-dicyano-2-imidazolyl)oxoethyl acrylate (16)}

Into a $100-\mathrm{mL}$ round-bottomed flask were placed 1 (2.04 g, $13.6 \mathrm{mmol})$, 2-hydroxyethyl acrylate $(2.01 \mathrm{~g}, 17.3 \mathrm{mmol})$, potassium carbonate $(1.85 \mathrm{~g}$, 
<smiles>Cn1c(F)nc(C#N)c1C#N</smiles><smiles>O=C(O)CCCCC(=O)O</smiles>

Scheme 1

$13.4 \mathrm{mmol})$, and THF (15 mL). The mixture was refluxed at $70{ }^{\circ} \mathrm{C}$ for $18 \mathrm{~h}$. The mixture was cooled to room temperature and poured into ice water $(50 \mathrm{~mL})$. The product was extracted with ethyl acetate $(20 \mathrm{~mL} \times 3)$. The ethyl acetate layer was washed with brine $(40 \mathrm{~mL} \times 2)$ and dried over anhydrous magnesium sulfate. The ethyl acetate was removed using a rotavapor to give a brown oily product $(2.2 \mathrm{~g})$. It was purified by column chromatography using ethyl acetate/hexane (1/3 v/v) mixture as an eluent ( $0.73 \mathrm{~g}, 23 \%$ yield). TLC $\mathrm{R}_{\mathrm{f}} 0.67$ (in EtOAc); MS (EI/70eV) $\mathrm{m} / z: 246$ (6, $\mathrm{M}+$ ), 174 (37), 99 (15), 67 (10), 55 (100), 40 (9), 27 (38), 18 (45); FTIR (KBr) 2960, 2235, 1727, 1562, 1494, 1409, 1324, 1297, 1271, $1182 \mathrm{~cm}^{-1} ;{ }^{1} \mathrm{H}$ NMR $\left(\mathrm{CDCl}_{3}\right) \delta(\mathrm{ppm}) 6.40(\mathrm{dd}, 1 \mathrm{H},=\mathrm{CHH}, J$ $=1,4 \mathrm{~Hz}, 17 \mathrm{~Hz}), 6.10(\mathrm{dd}, 1 \mathrm{H},=\mathrm{CH}-, J=10$ $\mathrm{Hz}, 17 \mathrm{~Hz}), 5.86(\mathrm{dd}, 1 \mathrm{H},=\mathrm{CHH}, J=1,4 \mathrm{~Hz}, 10$ $\mathrm{Hz}), 4.67\left(\mathrm{t}, 2 \mathrm{H}, \mathrm{OCH}_{2}, J=4.4 \mathrm{~Hz}\right), 4.49(\mathrm{t}, 2 \mathrm{H}$,
$\left.\mathrm{OCH}_{2}, J=4.4 \mathrm{~Hz}\right), 3.56\left(\mathrm{~s}, 3 \mathrm{H}, \mathrm{ArCH}_{3}\right) ;{ }^{13} \mathrm{C} \mathrm{NMR}$ $\left(\mathrm{CDCl}_{3}\right) \delta(\mathrm{ppm}) 155.7,153.6,131.7,127.8,118.2$, 111.9, 109.5, 108.3, 69.7, 61.7, 30.9; ANal. Calcd for $\mathrm{C}_{11} \mathrm{H}_{10} \mathrm{~N}_{4} \mathrm{O}_{3}$ : C, 53.66; H, 4.09. Found: C, 53.32; H, 4.02 .

\section{Poly[2-(1-methyl-4,5-dicyano-2-imidazolyl)oxoethyl acrylate] (17)}

Into a flame-dried 25-mL test tube with a magnetic stirrer and a rubber septum were placed 2-(1-methyl-4,5-dicyano-2-imidazolyl)oxoethyl acrylate $(\mathbf{1 6}, 0.30 \mathrm{~g}, 1.2 \mathrm{mmol})$, AIBN ( $4.0 \mathrm{mg}, 0.024$ $\mathrm{mmol})$, and toluene (4 mL). Nitrogen was bubbled through the solution for $30 \mathrm{~min}$. The mixture was stirred at $65{ }^{\circ} \mathrm{C}$ for $23 \mathrm{~h}$. Polymer separated from solution and coated the test tube. It was dissolved in acetone $(2 \mathrm{~mL})$ and was poured into ether $(50$ $\mathrm{mL})$. The white precipitate was dried in a vacuum oven $(0.24 \mathrm{~g}, 77 \%$ yield). FTIR (KBr) 2960, 2236, $1739,1702,1572,1558,1494,1452,1400,1356$, $1325,1249,1200,1174,1162,1118,1062,949$, $723 \mathrm{~cm}^{-1} ;{ }^{1} \mathrm{H}$ NMR (acetone- $\left.\mathrm{d}_{6}\right) \delta(\mathrm{ppm}) 4.74(\mathrm{~s}$, $\left.2 \mathrm{H}, \mathrm{OCH}_{2}\right), 4.49\left(\mathrm{~s}, 2 \mathrm{H}, \mathrm{OCH}_{2}\right), 3.71(\mathrm{~s}, 3 \mathrm{H}$, $\left.\mathrm{ArCH}_{3}\right), 2.44$ (s, br, 1H, CH), 2.0-1.4 (m, br, 2H, main chain $\mathrm{CH}_{2}$ ); Molecular weight by GPC $M_{n}$ $=28,000, M_{w}=58,000 ;$ ANAL. Calcd for

Table I. The Reaction Yield, UV-VIS and CV Data of the 2-Alkoxy and 2-Aryloxy Derivatives of 1-Methyl-4,5-dicyanoimidazole

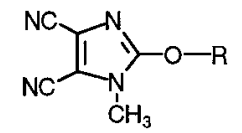

\begin{tabular}{|c|c|c|c|c|c|c|}
\hline \multirow[b]{2}{*}{$\mathrm{R}$} & \multirow[b]{2}{*}{ Product } & \multicolumn{2}{|c|}{ UV-VIS } & \multicolumn{2}{|c|}{ Cyclic Voltammetry } & \\
\hline & & $\lambda_{\max }$ & $\varepsilon$ & $\mathrm{V}_{\text {red }}(\mathrm{V})$ & Reversibility & \\
\hline$-\mathrm{CH}_{2} \mathrm{CH}_{3}$ & 2 & 271.0 & 11,932 & 2.64 & Yes (Quasi) & \\
\hline$-\mathrm{CH}_{2} \mathrm{CF}_{3}$ & 3 & 263.0 & 19,493 & 2.48 & Yes (Quasi) & \\
\hline$-\mathrm{CH}_{2}-\mathrm{C} \equiv \mathrm{CH}$ & 4 & 265.5 & 15,903 & 2.28 & No & \\
\hline & 5 & 268.5 & 12,143 & 2.59 & Yes (Quasi) & \\
\hline & 6 & 269.5 & 23,345 & 1.51 & Yes (Quasi) & \\
\hline & & & & 2.60 & No & \\
\hline & 7 & 90 & 267.5 & 15,708 & 2.42 & No \\
\hline & 8 & 93 & 268.5 & 16,373 & 2.42 & No \\
\hline
\end{tabular}




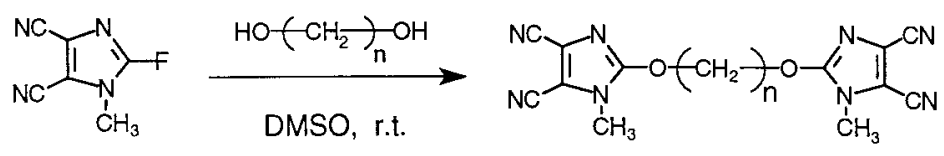

Scheme 2

$\mathrm{C}_{11} \mathrm{H}_{10} \mathrm{~N}_{4} \mathrm{O}_{3}$ : C, 53.66; H, 4.09. Found: C, 53.45; $\mathrm{H}, 4.18$.

\section{2-(1-Methyl-4,5-dicyano-2-imidazolyl)oxoethyl vinyl ether (18)}

Into a solution of 1 ( $1.30 \mathrm{~g}, 8.64 \mathrm{mmol})$ in DMSO (3 mL) were added 2-hydroxyethyl vinyl ether $(1.16 \mathrm{~g}, 13.2 \mathrm{mmol})$ and potassium carbonate $(0.683 \mathrm{~g}, 4.95 \mathrm{mmol})$. The mixture was stirred at room temperature for $22 \mathrm{~h}$. It was poured into ice water $(100 \mathrm{~mL})$ and the precipitate was collected by filtration. It was purified by column chromatography using ethyl acetate/hexane $(1 / 5 \mathrm{v} / \mathrm{v})$ as an eluent (1.62 g, 86\%). TLC $R_{\mathrm{f}} 0.65$ (in EtOAc); FTIR (KBr) 2231, 1622, 1566, 1496, 1449, 1400, 1360, 1327, 1201, 1181, 1064, 983, 963, $838 \mathrm{~cm}^{-1}$; ${ }^{1} \mathrm{H} \mathrm{NMR}\left(\mathrm{CDCl}_{3}\right) \delta(\mathrm{ppm}) 6.48(\mathrm{dd}, 1 \mathrm{H},=\mathrm{CH}-, J$ $=14 \mathrm{~Hz}, 7 \mathrm{~Hz}), 4.70\left(\mathrm{t}, 2 \mathrm{H}, \mathrm{OCH}_{2}, J=4.5 \mathrm{~Hz}\right)$, $4.23(\mathrm{dd}, 1 \mathrm{H},=\mathrm{CHH}, J=14 \mathrm{~Hz}, 2.5 \mathrm{~Hz}), 4.11(\mathrm{dd}$, $1 \mathrm{H},=\mathrm{CHH} J=7 \mathrm{~Hz}, 2.5 \mathrm{~Hz}), 4.05\left(\mathrm{t}, 2 \mathrm{H}, \mathrm{OCH}_{2}\right.$, $J=4.5 \mathrm{~Hz}$ ), 3.60 (s, 3H, $\mathrm{ArCH}_{3}$ ); Anal. Calcd for $\mathrm{C}_{10} \mathrm{H}_{10} \mathrm{~N}_{4} \mathrm{O}_{2}: \mathrm{C}, 55.04 ; \mathrm{H}, 4.62$. Found: C, 54.90; $\mathrm{H}, 4.60$.

\section{Poly[2-(1-methyl-4,5-dicyano-2-imidazolyl)oxoethyl vinyl ether] (19)}

Into a flame-dried 20-mL test tube sealed with a rubber septum was added a solution of $\mathbf{1 8}(0.35 \mathrm{~g}$, $1.6 \mathrm{mmol})$ in distilled toluene $(5 \mathrm{~mL})$. The test tube was chilled in a dry ice/acetonitrile bath to $-40{ }^{\circ} \mathrm{C}$. Then, $0.5 \mathrm{~mL}$ of an initiator stock solution made of boron trifluoride diethyletherate $(0.109 \mathrm{~g}, 0.768 \mathrm{mmol})$ and toluene $(5 \mathrm{~mL})$ was added to the test tube. The mixture was kept in the dry ice bath for $3 \mathrm{~h}$, and then slowly warmed to room temperature. The mixture was poured into hexane $(20 \mathrm{~mL})$ and the product precipitated. (0.06 g, 17\% yield); Molecular weight by GPC $M_{n}$ $=3900, M_{w}=5800$.

\section{RESULTS AND DISCUSSION}

\section{Syntheses of Alkoxy and Aryloxy Derivatives and Their CV}

In general, alcohols are less nucleophilic than amines, and do not work well in NAS reactions with 1-methyl-2-bromo-4,5-dicyanoimidazole. However, the development of 1-methyl-2-fluoro-4,5-dicyanoimidazole $(\mathbf{1})^{9}$ provided a new substrate for the synthesis of the 2-oxo derivatives. A fluoro substituent enhances the rate of substitution reaction compared to chloro, bromo, and iodo substituents. ${ }^{10}$ Various aliphatic and aromatic alcohols react with 1 to give the 2-alkoxy and aryloxy derivatives as shown in Scheme 1. The reactions were slower than those of amines but gave good yields after reflux.

The UV-VIS spectroscopic results of the derivatives are summarized in Table I. The absorptions are observed at shorter wavelength by 20-25 $\mathrm{nm}$ than those of the 2-dialkylamino derivatives $\left(\lambda_{\max }=285-295 \mathrm{~nm}\right)^{4(\mathrm{c}), 11}$, suggesting that delocalization of $\pi$-electrons, due to $\mathrm{p}-\pi^{*}$ interaction with the oxygen is weaker than that with nitrogen.

Cyclic voltammetry results for the derivatives are also listed in Table I. The alkoxy derivatives $(\mathbf{2}, \mathbf{3})$ except the propargyloxy derivative (4) show quasi-reversible cyclic voltammograms, as do the 2-dialkylamino derivatives discussed previously. ${ }^{5}$ The prefix "quasi" is used because the oxidation peak current is typically smaller than the reduction peak current. The irreversibility of $\mathbf{4}$ is likely due to the acidic acetylenic hydrogen, which can react with an electron during reduction. The phenoxy derivative (5) also shows a quasi-reversible cyclic voltammogram but no dimeric aryloxy derivatives $(\mathbf{7}, \mathbf{8})$ show signs of reoxidation. The $p$-nitrophenoxy derivative (6) showed a quasi-re-

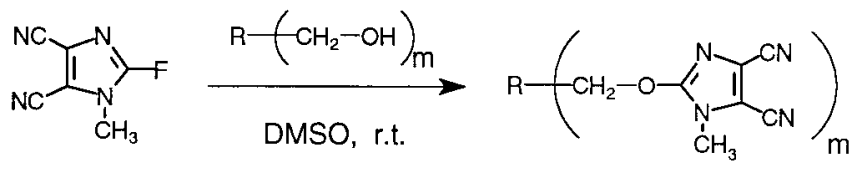

Scheme 3 

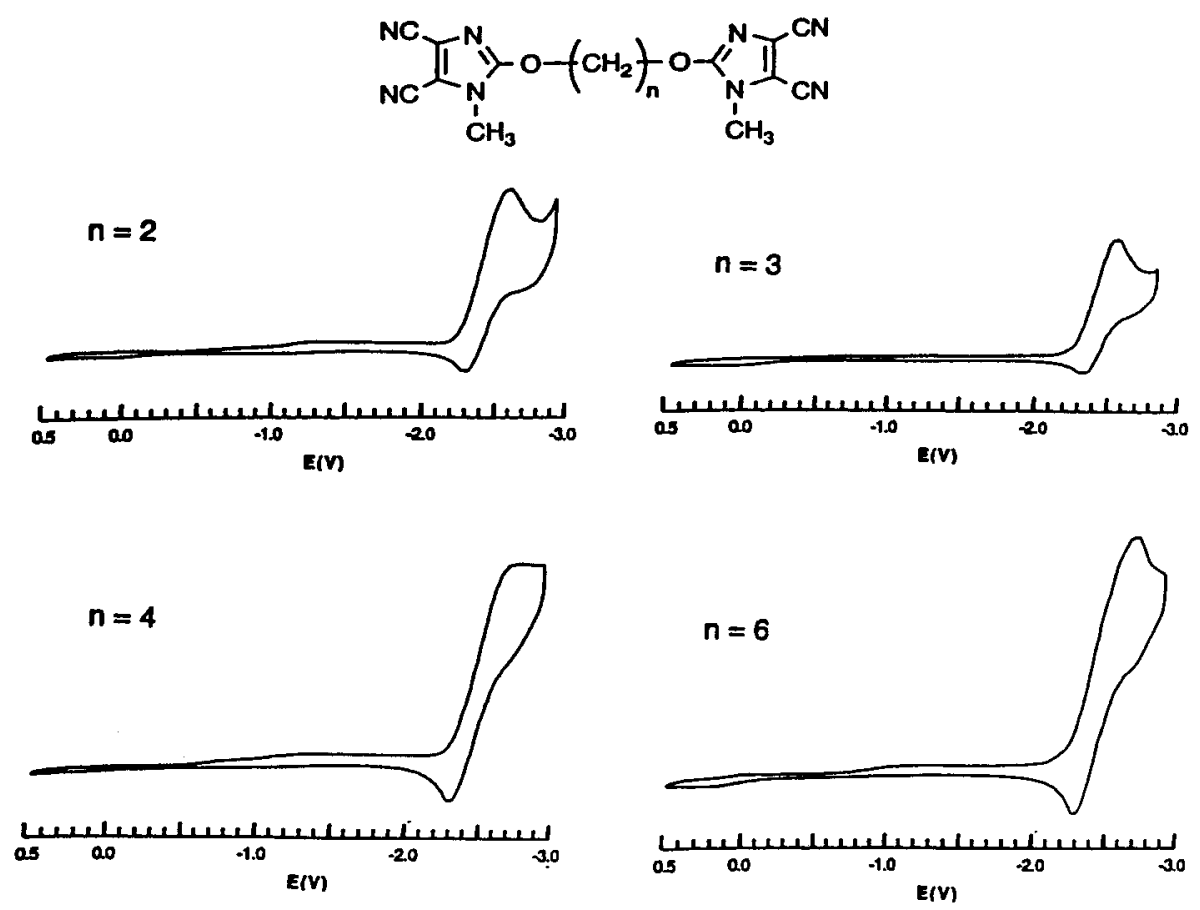

Figure 1. Cyclic voltammograms of alkoxy dimers with different lengths of alkyl chains between two dicyanoimidazoles (in $0.1 \mathrm{M} \mathrm{Et}_{4} \mathrm{NBF}_{4}$ /acetonitrile using Pt working electrode and silver reference electrode, scan rate $100 \mathrm{mV} / \mathrm{s}$ ).

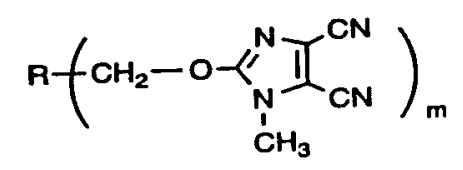
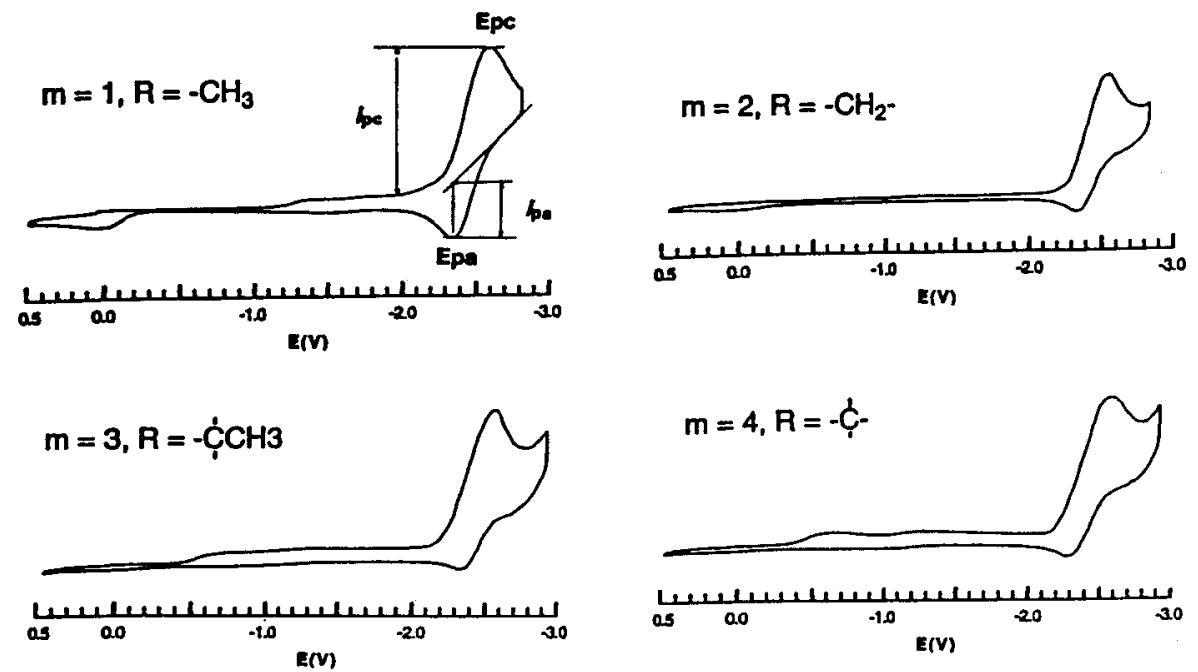

Figure 2. Cyclic voltammograms of alkoxy monomer to tetramer (in $0.1 M \mathrm{Et}_{4} \mathrm{NBF}_{4}$ in acetonitrile using $\mathrm{Pt}$ working electrode and silver reference electrode, scan rate 100 $\mathrm{mV} / \mathrm{s})$. 

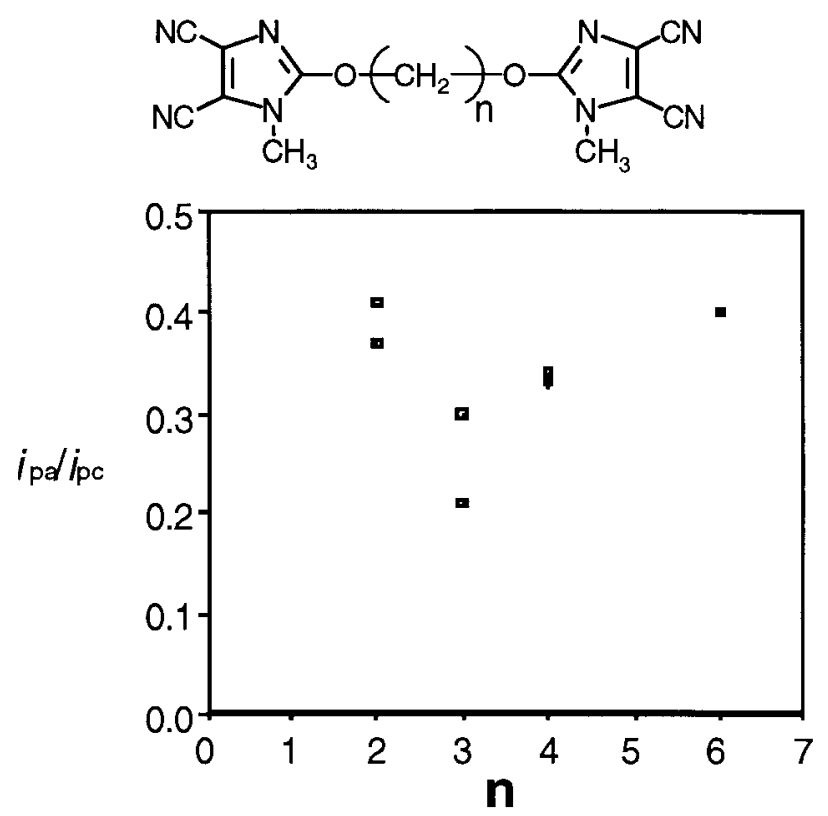

(a)
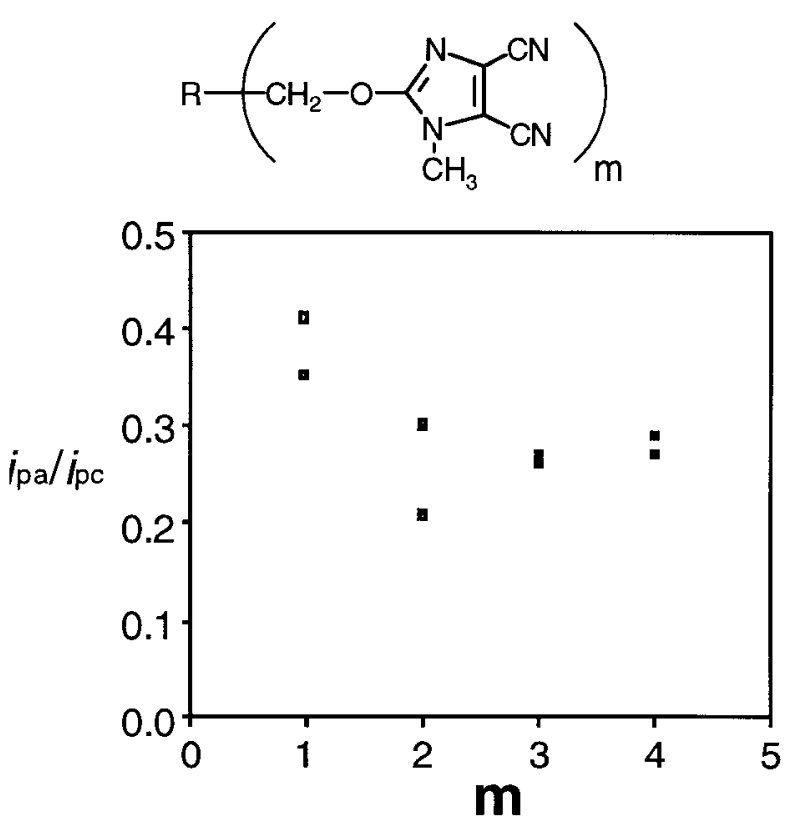

(b)

Figure 3. (a) The effect of spacer length, $n$, and (b) dicyanoimidazole density, $m$, on the degree of quasi reversibility $\left(i_{\mathrm{pa}} / i_{\mathrm{pc}}\right)$ of the alkoxy dicyanoimidazole model compounds.

versible wave for the reduction of nitrophenyl group at $1.5 \mathrm{~V}\left(\mathrm{vs} \mathrm{Ag} / \mathrm{Ag}^{+}\right.$) but not for the reduction of dicyanoimidazole at $2.6 \mathrm{~V}$.

\section{Oligomeric Model Compounds}

Dimers with various length of alkyl chains between two dicyanoimidazoles were synthesized by NAS reactions of $\mathbf{1}$ and aliphatic diols. Unlike the monosubstitution reactions, the dimer syntheses in THF with $\mathrm{K}_{2} \mathrm{CO}_{3}$ at reflux temperature yielded both the desired products and substantial side product, 1-methyl-3-(1-methyl-4,5-dicyano-2-imidazolyl)-4,5-dicyano-2-imidazolone. It has been observed previously that reactions involving $\mathbf{1}$ with oxyanions such as potassium carbonate can produce the imidazolone. ${ }^{9}$

Boswell et al. ${ }^{12}$ reported that activated aryl fluorides can undergo NAS reaction with alcohols in DMSO even at room temperature with high yields. Accordingly, the dimer reactions were run at room temperature in $\mathrm{DMSO}$ without $\mathrm{K}_{2} \mathrm{CO}_{3}$, as shown in Scheme 2. The yields were $30-50 \%$ after recrystallization without formation of the imidazolone.

Another series of compounds were prepared to investigate the effect of proximity of dicyanoimidazole molecules on their electrochemical properties. They include a single alkoxy up to tetramer with a three carbon separation between dicyanoimidazole units. They were synthesized by NAS reactions of $\mathbf{1}$ with various polyols as shown in Scheme 3.

\section{Effect of Spacer Length and Dicyanoimidazole Proximity}

Cyclic voltammograms of dimers with different spacer length are shown in Figure 1 and all are quasi-reversible. The degree of reversibility, ${ }^{13}$ as represented by the ratio of anodic peak current to cathodic peak current, $i_{\mathrm{pa}} / i_{\mathrm{pc}}$, was calculated and its correlation with the alkyl spacer length between dicyanoimidazole end units is plotted in Figure 3(a). Cyclic voltammograms were run twice for each sample, and the two data points are recorded in the graph. We expected that we could increase the degree of quasi-reversibility if we could decrease the chance for the dicyanoimidazole units to meet by introducing alkyl spacers. This appears to be the case except for the case of the ethylene spacer $(\mathbf{9})$. The dimer 9 showed a relatively high degree of quasi-reversibility. Perhaps the ethylene group, which is more rigid, prevents the two terminal dicyanoimidazoles from colliding with each other.

Cyclic voltammograms of monomer through tetramer are shown in Figure 2. They are also all 


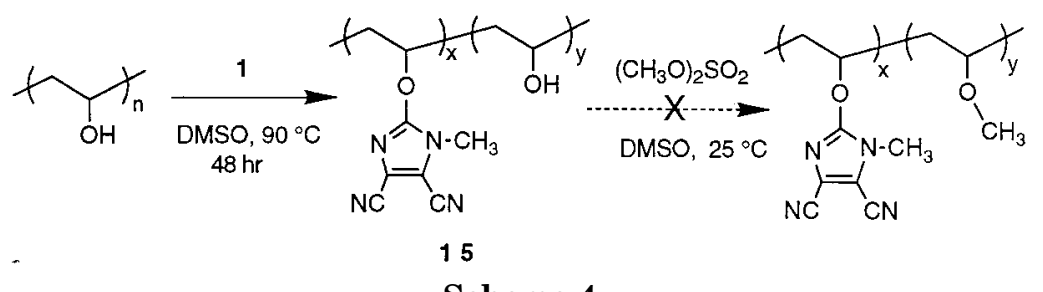

Scheme 4

quasi-reversible. Figure 3(b) shows the effect of dicyanoimidazole proximity on the degree of quasi-reversibility. Although the $i_{\mathrm{pa}} / i_{\mathrm{pc}}$ of monomeric derivative (2) stays in the range of $0.3-0.4$, those of dimer through tetramer $(\mathbf{1 0}, \mathbf{1 3}, \mathbf{1 4})$ fall to $0.2-0.3$.

\section{Polymer Substitution Reactions on Poly(vinyl alcohol)}

Poly(vinyl alcohol) is a readily available polymer that participates in a number of chemical modification reactions. The modified poly(vinyl alcohol)s find applications in the area of polymer catalysts, ${ }^{14}$ membranes, and medical polymers. ${ }^{15}$ To incorporate dicyanoimidazoles on a polymer chain and study its properties, a substitution reaction on poly(vinyl alcohol) was carried out with large excess of 1 in DMSO, as shown in Scheme 4. Although the NAS reactions of aliphatic alcohols and 1 gave relatively high yields as described above, the polymer gave incomplete substitution even at elevated temperature. An ${ }^{1} \mathrm{H}$ NMR study showed the degree of substitution is about $20 \%$.

The partially substituted polymer $\mathbf{1 5}$ was coated on a Pt electrode and a CV was run in acetonitrile- $\mathrm{Et}_{4} \mathrm{NBF}_{4}$. The cyclic voltammogram showed a reduction wave at $-2.7 \mathrm{~V}$ but no reversibility, probably because of unsubstituted hydroxy groups reacting with an electron. A methylation reaction using dimethylsulfate was attempted to remove any acidic protons in the polymer 15. However, the IR spectrum of the product still indicated the presence of a hydroxy group, and showed that the reaction was not complete.

\section{Polymers from Vinyl Monomers}

One way to get a $100 \%$ substituted polymer is to make a polymer starting with a substituted monomer. The acrylate group, one of the most facile polymerization groups, was successfully introduced onto 1-methyl-4,5-dicyanoimidazole with an oxygen linker by the reaction of 2-hydroxyethyl acrylate and $\mathbf{1}$, as shown in Scheme 5. The acrylate monomer $\mathbf{1 6}$ was polymerized by the radical initiator AIBN in toluene. The resulting polymer $\mathbf{1 7}$ has good solubility in most organic solvents and the molecular weight $\left(M_{w}\right)$ determined by GPC was 58,000.

A vinyl ether monomer with pendent dicyanoimidazole was also synthesized by a reaction of 2-hydroxyethyl vinyl ether and $\mathbf{1}$ as shown in Scheme 6. This monomer was polymerized in toluene using $\mathrm{Et}_{2} \mathrm{OBF}_{3}$ as a cationic initiator. The polymer had a molecular weight $\left(M_{w}\right)$ of 5800 . This polymer has an interesting structure in which two adjacent dicyanoimidazoles are connected by an ether linkage. Because crown ethers are known to bind cations, our hope was that the ether linkage would stabilize the counter cation of the reduced dicyanoimidazole moiety.

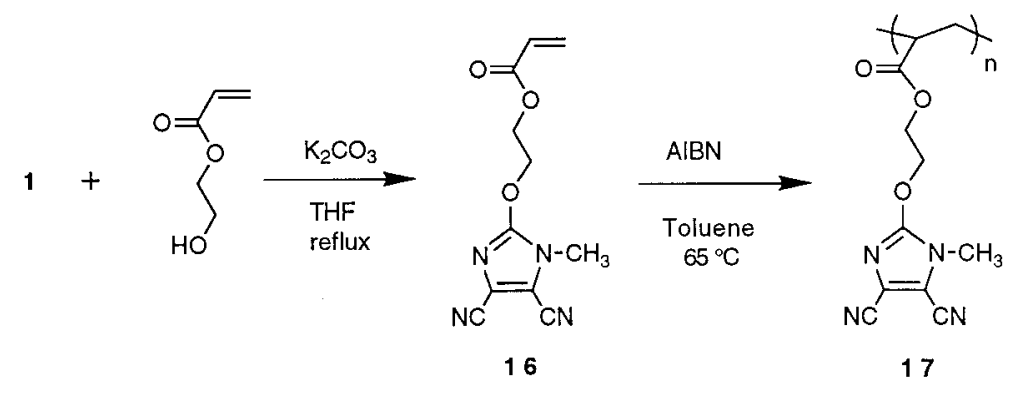

Scheme 5 


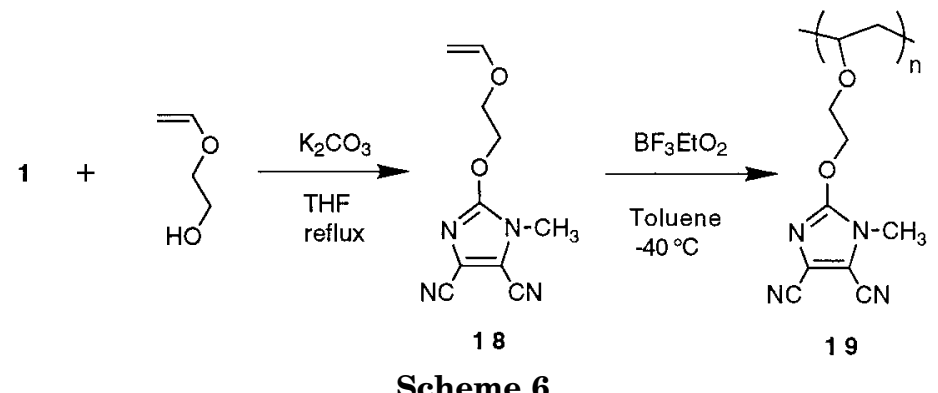

Scheme 6

CV studies were carried out on the polymers $\mathbf{1 7}$ and 19 dissolved in eletrolyte solution. However, the polymers did not have the reversible reduction behavior that had been observed for the monomeric and oligomeric compounds. Apparently intramolecular reactions between the reduced dicyanoimidazoles are simply too facile in the polymer.

\section{CONCLUSIONS}

We have reported the synthesis of several new 2-oxo derivatives of 1-methyl-4,5-dicyanoimidazoles and incorporation of this functional group into polymers in an effort to prepare electronaccepting material. The substitution of an electron-donating group such as alkoxy group at the 2-postion of 1-methyl-4,5-dicyanoimidazole leads the derivatives to be electrochemically quasi-reversible under redox conditions in acetonitrile. Their reduction potentials are from $-2.6 \sim-2.7 \mathrm{~V}$ vs $\mathrm{Ag} / \mathrm{Ag}^{+}$, implying that they are moderate organic acceptors. These results are in accord with our previous observations for the 2-dialkylamino derivatives of 1-methyl-4,5-dicyanoimidazole. On the other hand, the polymers with pendent dicyanoimidazole showed reduction waves with little reversibility. The CV study of oligomeric model compounds showed that reversibility of the dicyanoimidazole compounds decreases as the reduced moieties are crowded together, perhaps because of intramolecular reactions between the reduced dicyanoimidazoles. The syntheses of these new cyanoimidazole pendent polymers do offer attractive possibilities for further work. Because cyano groups are easily hydrolized to carboxylic acids or reduced to amines, polymers with high density of functional groups can easily be prepared. These apects of pendent cyanoimidazole polymers are under further investigation.

\section{REFERENCES AND NOTES}

1. (a) Cowan, D. O.; Wlygul, F. M. Chem Eng News 1986, July 21, 28; (b) Hertler, W. R. J Org Chem 1976, 41, 1412.

2. Martin, N.; Segura, J. L.; Seoane, C. J Mater Chem 1997, 7, 1661.

3. (a) deLeeuw, D. M.; Simenon, M. M. J.; Brown, A. R.; Einerhand, R. E. F. Synth Met 1997, 87, 53;

(b) Arbizanni, C.; Mastragostino, M.; Meneghello, L.; Paraventi, R. Adv Mater 1996, 8, 331.

4. Skotheim, T. A., Ed. Handbook of Conducting Polymers; Marcel Dekker: New York, 1986; (b) Scranton, A. B.; Rangarajan, B.; Klier, J. In Advances in Polymer Science; Springer-Verlag: Berlin, 1995, p. 1, vol. 122; (c) MacDiarmid, A. G.; Epstein, A. J Chem Soc, Faraday Trans, 1989, 5, 1; (d) Miller, J. S. Adv Mater 1993, 5, 671; (e) Bäurele, P. Adv Mater 1993, 5, 879.

5. (a) Wan, M. X.; Arbuckle, G. A.; MacDiarmid, A. G. Synth Met 1988, 24, 283; (b) Aeiyach, S.; Soubiran, P.; Lacaze, P. C. Synth Met 1989, 32, 103; (c) Mastragostino, M.; Soddu, L. Electrochim Acta 1990, 35, 463; (d) Steinmüller, D. Phys Rev B 1993, 47, 13323; (e) Rudge, A.; Raistrick, I.; Gottesfeld, S.; Ferraaris, P. Electrochim Acta 1994, 39, 273; (f) Zotti, G.; Schiavon, G. Synth Met 1994, 63, 53; (g) Ferraris, J. P.; Henderson, C.; Torres, D.; Meeker, D. Synth Met 1995, 72, 147; (h) Yamamoto, T.; Maruyama, T.; Zhou, Z.-H.; Ito, T. J Am Chem Soc 1994, 116, 4832; Yamamoto, T. J Polym Sci Part A Polym Chem 1996, 34, 997.

6. (a) Allan, D. S.; Bergstrom, D. F.; Rasmussen, P. G. Synth Met 1988, 25, 139; (b) Allan , D. S.; Thurber, E. L.; Rasmussen, P. G. J Polym Sci Part A Polym Chem 1990, 28, 2475; (c) Apen, P. G.; Rasmussen, P. G. J Am Chem Soc 1991, 113, 6178.

7. (a) Jang, T.; Rasmussen, P. G. Polym Preprints 1994, 35, 663; (b) Jang, T.; Rasmussen, P. G. Polym Preprints 1995, 36, 245; (c) Jang, T.; Rasmussen, P. G. J Polym Sci Part A Polym Chem 1998, 36, 2619.

8. Subrayan, R. P.; Rasmussen, P. G. Tetrahedron $1995,51,6167$. 
9. Coad, E. C. Ph.D. Thesis, The University of Michigan, Ann Arbor, 1994.

10. Terrier, F. In Nucleophilic Aromatic Displacement; VCH Publishers, New York, 1991, p. 1.

11. Subrayan, R. P. Ph.D. Thesis, The University of Michigan, Ann Arbor, 1993.

12. Boswell, G. E.; Licause, J. F. J Org Chem 1995, 60, 6592. For other NAS reactions on aryl fluoride compounds, see (a) Feldman, D; Segal-Lew, D.; Rabinovitz, M. J Org Chem 1991, 56, 7350; (b) Koch, A. S.; Feng, A. S.; Hopkins, T. A.; Streitwiesser, A.J Org Chem 1993, 58, 1409; (c) Persson, J.; Axelsson, S.; Matsson, O. J Am Chem Soc 1996, 118, 20; (d)
Subrayan, R. P.; Kampf, J. W.; Rasmussen, P. G. J Org Chem 1996, 59, 4341.

13. For nomenclature and general concept for cyclic voltammetry, see (a) Evans, D. H.; O'Connell, M. K.; Petersen, R. A.; Kelly, M. J. J Chem Ed 1983, 60, 290; (b) Rusling, J. F.; Suib, S. L. Adv Mater 1994, 6, 992; Gosser, K. K., Jr. Cyclic Voltammetry; VCH Publishers Inc.: New York, 1994.

14. Hrudkova, H.; Cefelin, P.; Janout, V. Collect Czech Chem Commun 1987, 52, 2204.

15. Li, F.; Wang, L.; Feng, X Gaofenzi Xuebao 1987, 1, 13. See also Chem Abstr 107161604. 